OPEN ACCESS

Edited by:

Jorge Blanco,

Universidade de Santiago de

Compostela, Spain

Reviewed by:

James P. O'Gara,

NUI Galway, Ireland

Javier Fernández,

Central University Hospital of Asturias,

Spain

*Correspondence: Dafne Pérez-Montarelo

dafne_perez@hotmail.com

Specialty section: This article was submitted to

Infectious Diseases,

a section of the journal

Frontiers in Microbiology

Received: 16 February 2017 Accepted: 04 May 2017

Published: 19 May 2017

Citation:

Pérez-Montarelo D, Viedma E Murcia M, Muñoz-Gallego I,

Larrosa N, Brañas $P$

Fernández-Hidalgo N, Gavaldà J, Almirante B and Chaves F (2017)

Pathogenic Characteristics of Staphylococcus aureus Endovascular Infection Isolates from Different Clonal

Complexes. Front. Microbiol. 8:917. doi: 10.3389/fmicb.2017.00917

\section{Pathogenic Characteristics of Staphylococcus aureus Endovascular Infection Isolates from Different Clonal Complexes}

\author{
Dafne Pérez-Montarelo ${ }^{1 *}$, Esther Viedma ${ }^{1}$, Mercedes Murcia ${ }^{1}$, Irene Muñoz-Gallego ${ }^{1}$, \\ Nieves Larrosa ${ }^{2}$, Patricia Brañas ${ }^{1}$, Nuria Fernández-Hidalgo ${ }^{3}$, Joan Gavaldà ${ }^{3}$, \\ Benito Almirante ${ }^{3}$ and Fernando Chaves ${ }^{1}$

\footnotetext{
${ }^{1}$ Department of Microbiology, Instituto de Investigación Hospital de Octubre, Madrid, Spain, ${ }^{2}$ Department of Microbiology, Hospital Universitari Vall d'hebron, Universitat Autónoma de Barcelona, Barcelona, Spain, ${ }^{3}$ Department of Infectious Diseases, Hospital Universitari Vall d'hebron, Universitat Autónoma de Barcelona, Barcelona, Spain
}

Staphylococcus aureus is a major cause of bacteremia and, even with appropriate clinical management, causes high morbidity, and mortality due to its involvement in endovascular complications and metastatic infections. Through different pathogenic in vivo and in vitro models we investigated the behavior of $S$. aureus most relevant clonal complexes (CCs) causing endovascular complications. We analyzed $14 \mathrm{~S}$. aureus strains representing CC5, CC8, CC15, CC30, and CC45 that caused endovascular complications, including methicillin susceptible and resistant isolates and strains with different functionality of the agr global regulator. Their adherence to collagen, interaction with the endothelium, resistance to immune attack, capacity to form biofilm and virulence in the Galleria mellonella model were analyzed. CC30 and CC45 showed greater adhesion to collagen and CC8 showed a trend towards higher rate of intracellular persistence in endothelial cells. All CCs exhibited similar tolerance to neutrophil antimicrobial peptide hNP-1 and were capable of forming biofilms under static conditions. The virulence assay in the G. mellonella model demonstrated that $\mathrm{CC} 15$ and CC30 were the most and least virulent, respectively. The analysis of the genomic sequences of the most relevant virulence genes identified some CC15 specific gene patterns (absence of enterotoxins and sak gene) and variants (mainly in leucocidins and proteases), but did not reveal any gene or variant that could be responsible for the increased virulence detected for CC15 strains. Even though all the CCs were capable of causing endovascular complications, our results showed that different CCs are likely to produce these complications through different mechanisms which, if confirmed in more sophisticated models, would indicate the need to more specific management and therapeutic approaches.

Keywords: Staphylococcus aureus, clonal complex, virulence, endovascular complications 


\section{INTRODUCTION}

Staphylococcus aureus is the leading cause of both healthcareand community-associated bloodstream infections in the industrialized world and is associated with significant morbidity and mortality. S. aureus is an opportunistic pathogen that upon entry to the cardiovascular system can lead to serious complications, such as infective endocarditis or thrombophlebitis, resulting in organ failure and death. Previous studies have identified clinical factors that reduce the occurrence of these complications, like early and aggressive antibiotic therapy and removal of intravascular devices (Fowler et al., 2005; Naber, 2009). However, endovascular complications remain commonplace in spite of appropriate management and treatment (Naber, 2009) suggesting that the intrinsic pathogenicity of the $S$. aureus strains involved may play a role in determining clinical outcome and development of endovascular complications.

Although significant progress has been made in the understanding of molecular mechanisms leading to this type of infection investigating specific $S$. aureus genetic markers, much work remains. Moreover, previous reports seem to indicate that no single virulence factor alone is sufficient to describe $S$. aureus endovascular pathogenesis and a cumulative effect from different factors probably offers the most realistic scenario (Peacock et al., 2002; Bouchiat et al., 2015). S. aureus has a highly clonal population structure with CCs comprising closely related, although not identical, genetic backgrounds (Lindsay et al., 2006; Dayan et al., 2016). Therefore, the analysis of the pathogenic characteristics of representative $S$. aureus genetic clonal complexes seems an appropriate means by which further our understanding of the etiology of this disease. Indeed, previous studies showed that, although most $S$. aureus genotypes exhibit the capacity to cause invasive disease, bacteremia caused by strains belonging to particular clonal complexes (CC5, CC15, and CC30) has been associated with endovascular complications (Fowler et al., 2007; Nienaber et al., 2011; Bouchiat et al., 2015). Methicillin resistance has also been linked to an increased risk for the development of hematogenous complications (Fowler et al., 2005, 2007). Nevertheless in most cases, the determinants of these associations remain poorly understood.

Several traits have been previously associated with the occurrence of endovascular complications after $S$. aureus bacteremia. An early crucial event in the hematogenous infectious process is the adherence to the endothelium or matrix proteins. Studies in animal models suggested that the ability to interact with collagen, which provides structural support and is present in heart valves, aortic tissues and damaged endothelial tissues, could be advantageous in terms of $S$. aureus endovascular pathogenesis (Patti et al., 1994; Gillaspy et al., 1998). Similarly, the binding of S. aureus to endothelial cells, which form the inner lining of cardiovascular system blood vessels, results critical, and leads to impairment of vascular function (Garciarena et al., 2015). Moreover, S. aureus can invade and persist intracellularly in endothelial cells, hidden from the host immune system, and extracellular antimicrobial treatment (Proctor et al., 2006; Garzoni and Kelley, 2009; Xiong et al.,
2009; Sinha and Fraunholz, 2010). During the initial stages of endovascular pathogenesis, antimicrobial hNP-1 peptide family produced by neutrophils is an important host defense mechanism mediating S. aureus clearance (Ganz, 2003; Rigby and DeLeo, 2012). In addition, the ability to form biofilm is an important virulence factor in damaged host tissues (McCarthy et al., 2015).

Therefore, our aim was to characterize the pathogenic behavior of the most relevant $S$. aureus genetic backgrounds causing endovascular complications, using different in vivo and in vitro models in a collection of representative clinical strains that also allowed us to analyze the effect of methicillin resistance and functionality of the accessory gene regulator (agr) that controls virulence factors expression. These strains were analyzed to determine their efficiency of adhesion to collagen; their capacity to adhere to, invade and persist within endothelial cells; their susceptibility to the antimicrobial neutrophil peptide hNP-1; their capacity to form biofilm; as well as their global virulence in the Galleria mellonella in vivo model.

\section{MATERIALS AND METHODS}

\section{Selection of S. aureus Strains Causing Endovascular Complications}

$S$. aureus clinical strains causing endovascular complications were selected from those recovered in two Spanish multicenter studies that focused on complicated catheter-related bacteremia and infective endocarditis (Fernández-Hidalgo, 2016; San-Juan et al., 2016). These studies recorded each patient's clinical history and, most importantly, ensured that their infections were properly managed and treated. The studies were approved by the ethics committee of the Hospital Universitario 12 de Octubre (Madrid, Spain) that considered not necessary to obtain written informed consent because participants were anonymized. A total 259 strains recovered from blood cultures in these studies were processed and isolates were identified according to standard techniques. The strains were characterized for agr functionality by measuring $\delta$-hemolysin production as previously reported (Seidl et al., 2011). Genotypic characterization was performed by pulsed-field gel electrophoresis (PFGE) (Chaves et al., 2005), Multilocus Sequence Typing (MLST) (Enright et al., 2000), and DNA microarrays (Alere) (Monecke et al., 2007, 2008).

Following review of the genotypic and phenotypic characteristics of the whole collection, several S. aureus strains, that represent the most common CCs in our collection (CC30, CC5, CC45, and CC8), and those CCs previously associated with endovascular complications (CC5, CC15, and CC30), were selected for further analyses. We randomly selected two methicillin susceptible (MSSA) strains of each CC, trying to include strains with different functionality of the agr global regulator when possible. Moreover, methicillin resistant isolates (MRSA), belonging to these CCs were also selected if available (there were no CC30 and CC15 MRSA in our collection; two CC5 MRSA isolates were selected according to the microarrays results to represent ST5 and ST125 strains). The main clinical 
and microbiological characteristics of the total 14 S. aureus strains selected are presented in Table 1.

\section{Collagen Binding Assay}

The collagen binding assay was performed according to the method described by Waterhouse and Russell (2006). Briefly, plates were coated with $50 \mu \mathrm{g}$ collagen, incubated overnight at $4^{\circ} \mathrm{C}$, washed, blocked for $2 \mathrm{~h}$ at $37^{\circ} \mathrm{C}$ with $3 \%$ bovine serum albumin (BSA) in PBS and washed again. One milliliter of a $10^{3} \mathrm{CFU} / \mathrm{ml} \mathrm{S}$. aureus suspension was added to each well and incubated at $37^{\circ} \mathrm{C}$ for $2 \mathrm{~h}$. The number of $\mathrm{CFU}$ adhered to the collagen was determined after washing by pouring MuellerHinton agar and incubating overnight at $37^{\circ} \mathrm{C}$. Results were analyzed by comparing the number of $\mathrm{CFU}$ recovered to each particular initial inoculum concentration as determined by viable count.

\section{Adhesion, Invasion, and Intracellular Persistence to Endothelial Cells}

Endothelial cells were harvested from umbilical cord veins and cultured as previously described (Davis et al., 2007). Adhesion, invasion, and intracellular persistence assays were performed according to Richards et al. (2015). Ten milliliters of a $10^{9} \mathrm{CFU} / \mathrm{ml}$ S. aureus suspension were added to confluent endothelial cells. The inoculum concentration was confirmed for each experiment by serial dilution and plating. For each strain, three plates were incubated at $37^{\circ} \mathrm{C}$ in $5 \% \mathrm{CO}_{2}$ for $2 \mathrm{~h}$. To assess invasion and intracellular persistence, the culture medium from two plates was replaced with Endothelial Cell Growth Medium (EGM-2) with $200 \mu \mathrm{g} / \mathrm{ml}$ gentamicin and the plates were returned to the incubator for either a further $3 \mathrm{~h}$ (invasion) or $72 \mathrm{~h}$ (intracellular persistence). The third plate was used to analyze cellular adhesion.

At the appropriate time points for each test method, endothelial cells were lysed with $1 \%$ triton-X-100 and serial dilutions were prepared and plated to determine viable counts. The adherence, invasion and persistence rates for each strain were expressed as the average percentage $( \pm S D)$ of the initial inoculum recovered from at least three independent experiments.

\section{Susceptibility to Human Neutrophil Peptide 1 (hNP-1)}

Each strain's susceptibility to hNP-1 was assayed by exposing $10^{5} \mathrm{CFU} / \mathrm{ml}$ to $10 \mu \mathrm{g} / \mathrm{ml}$ of hNP-1 (Biogen Cientifica SL) in a solution containing $10 \mathrm{nM}$ potassium phosphate, $\mathrm{pH}$ 7.4, containing $1 \% \mathrm{BHIB}$, for $2 \mathrm{~h}$ at $37^{\circ} \mathrm{C}$ and processed for quantitative culture as previously described (Seidl et al., 2011). The results are expressed as the mean percentage $( \pm S D)$ of the initial inoculum that survived exposure to hNP-1. A minimum of three independent assays were performed for each strain.

\section{Biofilm Formation under Static Conditions}

Biofilm formation under static conditions was analyzed as previously described (Seidl et al., 2011). In brief, S. aureus was resuspended to a density of $1.5 \mathrm{McF}$ arland, diluted 1:100 in brain heart infusion broth (BHIB) supplemented with $0.2 \%$ glucose, incubated for $18 \mathrm{~h}$ at $37^{\circ} \mathrm{C}$, washed and stained with Safranin.

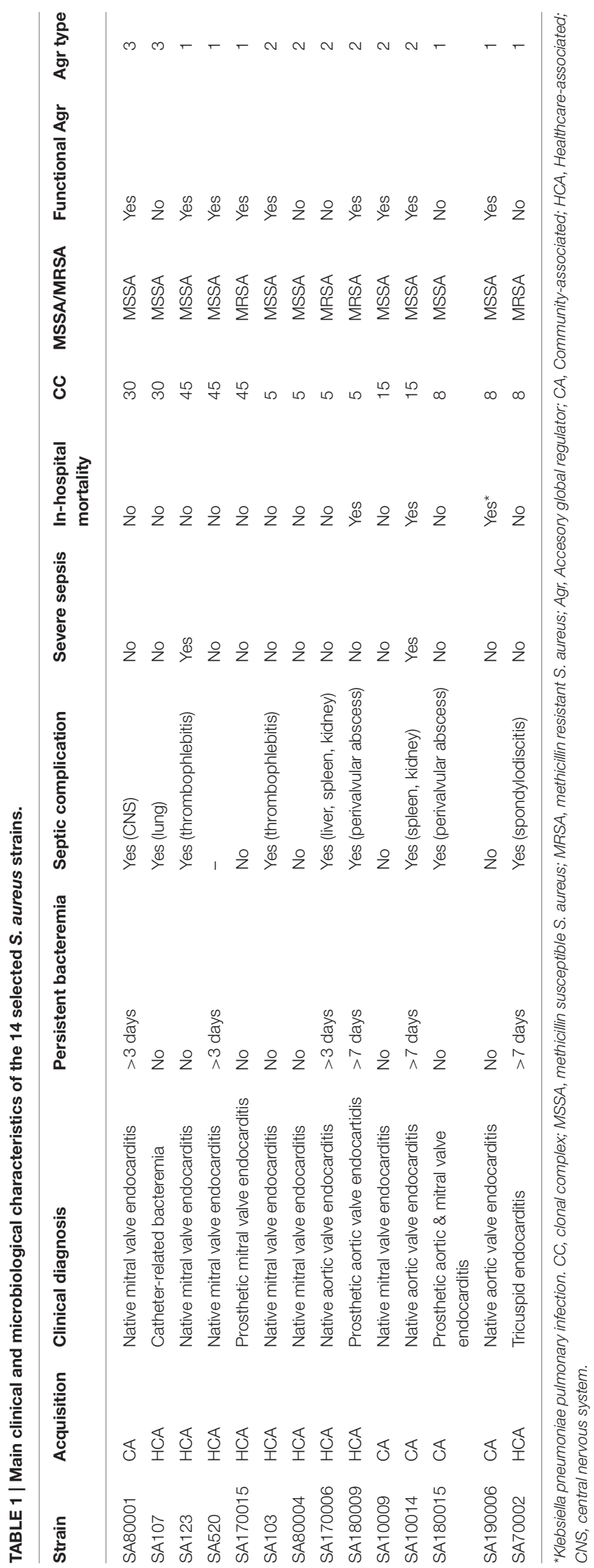


Absorbance was measured at $490 \mathrm{~nm}$ and a value $>0.5$ was considered indicative of biofilm formation (Seidl et al., 2011). Each strain was tested three times and the results are presented in terms of the minimum absorbance \pm standard deviation $(S D)$.

\section{Virulence Assay in the Galleria mellonella In vivo Model}

The invertebrate G. mellonella (Bichosa) infection model was used to study the virulence of the $S$. aureus strains as previously described (Peleg et al., 2009). To ensure that the observations in our study were not due to differences in growth rates, the growth kinetics of each strain were assessed by measuring the change in $\mathrm{OD}_{600}$ in broth cultures over $24 \mathrm{~h}$. Fifteen healthy caterpillars of similar size $(\sim 200 \mathrm{mg})$ were used in each assay and $10 \mu \mathrm{l}$ of a $10^{8} \mathrm{CFU} / \mathrm{ml}$ bacterial suspension were injected into the hemocoel of each caterpillar. After injection, caterpillars were incubated at $37^{\circ} \mathrm{C}$ and the number of dead caterpillars was recorded every $24 \mathrm{~h}$ over a period of 5 days. Dead larvae were identified as those melanised and unresponsive to stimuli. Bacterial colony counts were used to confirm all initial $S$. aureus inocula. Three control groups of larvae were included in each experiment, the first received no injection, the second was inoculated only with phosphate buffered saline (PBS) to monitor for killing due to physical trauma and the third was inoculated with a bacterial strain inactivated at $95^{\circ} \mathrm{C}$ for $10 \mathrm{~min}$, to discard killing by a noninfectious reaction due to $S$. aureus components. Experiments with more than two dead caterpillars in the control groups were aborted and repeated. At least three independent experiments were performed for all strains.

\section{Genomic Comparison of Relevant Virulence Genes}

Whole Genome sequencing of all strains was performed using TruSeq DNA PCR free kits (Illumina, CA) according to recommended procedures. Libraries were sequenced on a single run on Illumina MiSeq intrument (150 bp paired-end reads) to generate a coverage of $\sim 100 \mathrm{X}$, with $350 \mathrm{bp}$ of insert size. A total of $\sim 1$ million reads were obtained on average for each isolate (range $1,560,800-2,372,112$ ). Reads were quality-trimmed (Q30) and reviewed using fastx_trimmer and fastqc software version 0.10.1 (http://www.bioinformatics.babraham.ac.uk/projects/fastqc/), respectively. Resulting reads were assembled into contigs using SPAdes software (version 3.8, http://bioinf.spbau.ru/spades), and their quality was evaluated with QUAST (version 4.3, http://quast.sourceforge.net/quast). The number of contigs for each genome ranged from 52 to 222. The general characteristics of genomes sequencing and assembly are shown in Supplementary Table 1. This Whole Genome Shotgun project has been deposited at GenBank under the accession numbers MKYX00000000, MKYY00000000, MKYZ000000 00, MKZA00000000, MKZB00000000, MKZC00000000, MKZ D00000000 MKZE00000000, MKZF00000000, MKZG00000 000, MKZH00000000, MKZI00000000, MKZJ00000000, and MKZK00000000. Open reading frames (ORFs) were predicted and annotated with the Rapid Annotations using Subsystems Technology (RAST) server (Aziz et al., 2008). This server identified protein-encoding genes, assigned functions to the genes, and allowed us identifying the most relevant virulence genes and their positions. Additionally, VirulenceFinder website (https://cge.cbs.dtu.dk/services/VirulenceFinder/) was also used for virulence genes identification. The genetic sequences of the virulence genes identified were aligned and compared using the Geneious server (geneious 10.0.06) to identify CC15 specific variants through multiple alignments. We defined as CC15 specific variants those variants detected in CC15 strains that were not present in any other strain belonging to a different CC.

\section{Statistical Analyses}

Means and standard deviations for a minimum of three independent experiments were calculated and plotted for each assay. Continuous variables are expressed as mean and standard deviation, and univariate comparisons were made with the Student's $t$-test or Mann-Whitney's $U$-test, as appropriate. Categorical parameters are expressed as absolute number and percentage, and univariate comparisons were performed with the $X^{2}$ test, or Fisher's exact test, as appropriate. When significant differences were observed, subsequent stratified analyses were performed. For G. mellonella survival analysis, larvae mean survival curves were plotted using the Kaplan-Meier method and differences in survival rates between groups were calculated using the log-rank test. All tests were two-tailed, and a $p<0.05$ was considered statistically significant. Statistical analysis was performed with SPSS statistics software (version 15.0, SPSS, Chicago, IL, USA).

\section{RESULTS}

\section{CC30 and CC45 Strains Showed Adhesion to Collagen}

The results obtained for individual strains are shown in Supplementary Figure 1. Comparison of the percentage of bacterial cells bound to collagen between the different CCs showed that CC45 and CC30 had the highest capacity to bind collagen (18.6 and $18.9 \%$ inoculum recovery, respectively; Figure 1A) and that CCs 5, 8, and 15 exhibited almost no collagen binding. Significant differences among the different CCs were detected $(p=0.051)$. This could represent an advantage for CC45 and CC30 strains that would adhere easily to the collagen present at endovascular tissues. In contrast, no differences in collagen binding were detected between agr functional and dysfunctional strains (9.7 and 4.3\%, respectively; $p=0.898$; Figure 1B) or methicillin resistance phenotype (5.2 and $8.9 \%$ for MRSA and MSSA respectively, $p=0.5454$; Figure $1 \mathrm{C}$ ).

\section{CC8 Strains Presented the Highest Adhesion, Invasion, and Intracellular Persistence Rates to Endothelial Cells}

The results obtained for adhesion, invasion and intracellular persistence of the individual strains are shown in Supplementary Figure 2. The percentage of adhesion to endothelial cells, present at the inner lining of endovascular system vessels, varied among the different CCs and ranged 

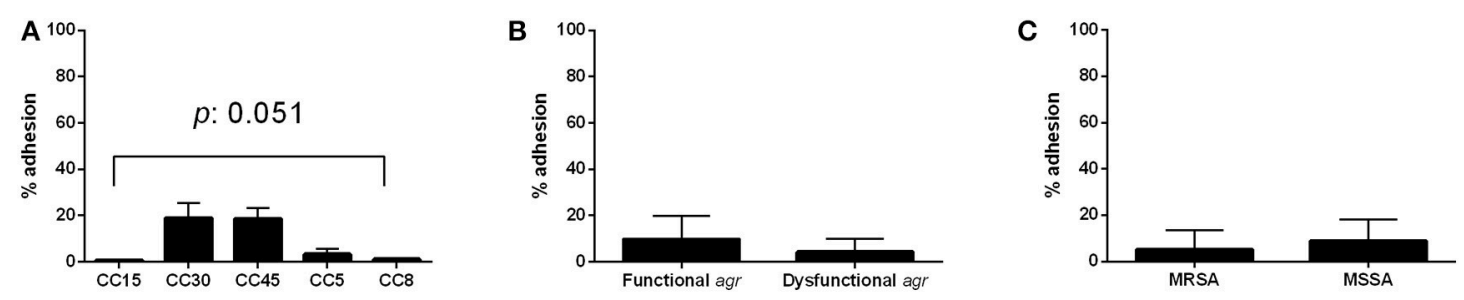

FIGURE 1 | Adherence to collagen. Adhesion percentage to collagen according to CC (A), agr functionality (B), and methicillin resistance (C). Bars and error bars represent the mean and standard deviation of at least 3 independent experiments. Only significant $p$-values are shown.
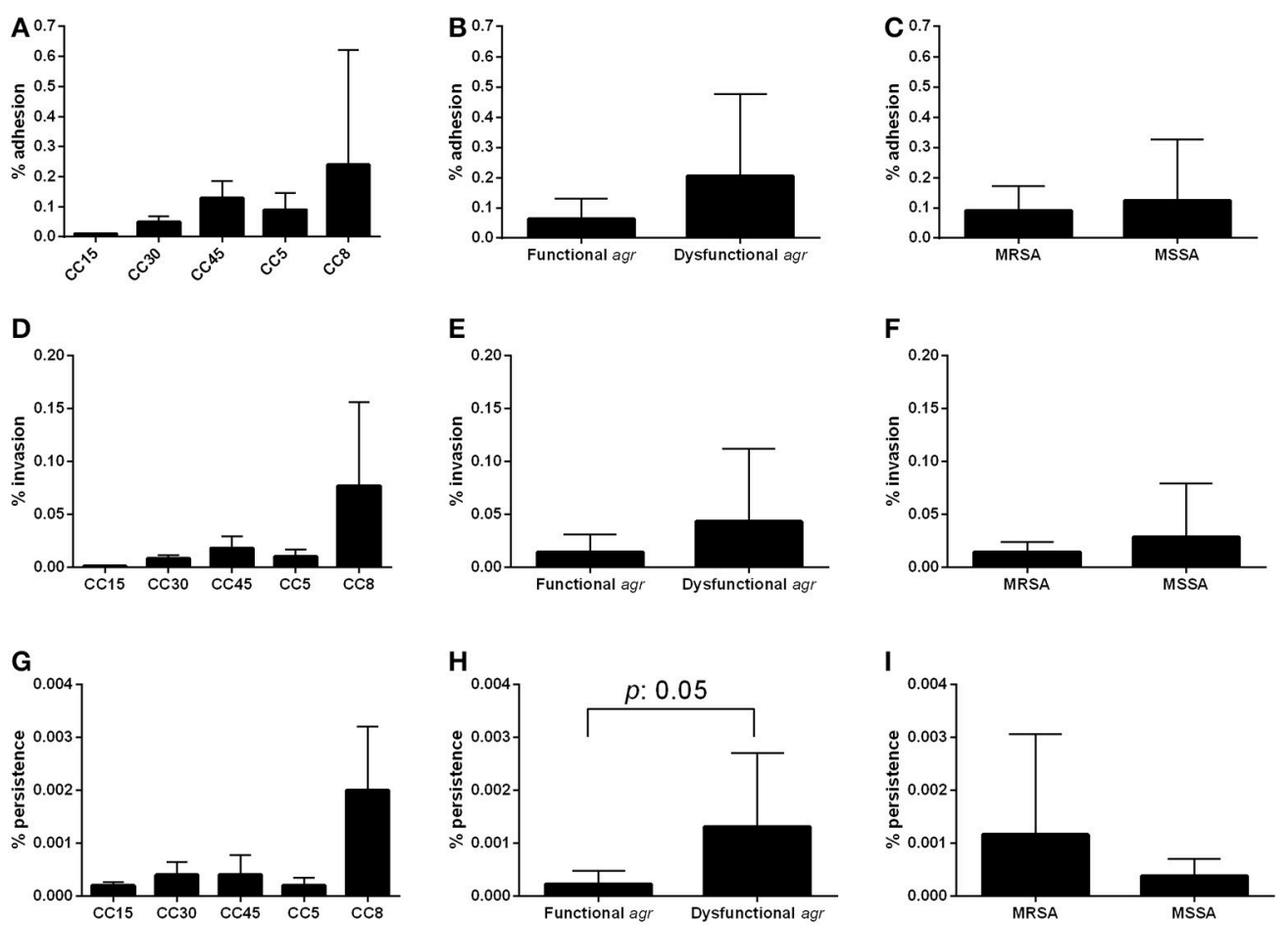

FIGURE 2 | Adhesion, invasion, and intracellular endothelial cells assay. Confluent endothelial cell obtained from umbilical cord veins were infected with $10^{7}$ CFU and the percentage of bacterial cells able to adhere (A-C), invade (D-F) and persist intracellularly (G-I) is represented according CC, agr functionality and methicillin resistance. Results are expressed as the percentage of the initial inoculum recovered. Bars and error bars indicate the mean and standard deviations of at least three independent experiments. Only significant $p$-values are shown.

between $0.005 \%$ for CC15 to $0.24 \%$ for CC8 (Figure $2 \mathrm{~A}$ ). It is necessary to take into account that, among the three CC8 strains included in the study, strain SA180015 showed high levels of adhesion and invasion (results for intracellular persistence were comparable to the other strains), which could distort the overall results for this group for both adhesion and invasion experiments. Additionally, although not statistically significant, a trend toward higher adhesion levels was observed for agr dysfunctional strains compared with agr functional strains $(p$ $=0.518$; Figure 2B). No differences in adhesion percentage were observed between strains of MRSA and MSSA ( $p=0.733$; Figure 2C).

A similar pattern was observed among the different CCs for the capacity to invade endothelial cells, with CC15 showing the lowest invasion rate and CC8 the highest, although the differences were not statistically significant (Figure 2D). Indeed, even if we do not consider SA180015, and consider only the other two CC8 strains (SA70002 and SA190006) CC8 is still the one showing the highest invasion percentage. The results observed for invasion when stratified by agr functionality and methicillin resistance were also not statistically significant and were comparable to those observed for adhesion $(p=0.438$ and $p=0.454$, respectively; Figures $2 \mathrm{E}, \mathbf{F})$.

In general, the pattern for intracellular persistence was similar to that observed for adhesion and invasion, being again CC8 strains the ones showing higher intracellular persistence rates, with no statistically significant differences between CCs. Nevertheless, the difference between CC8 and 
CC5 was almost significant ( $p=0.056$; Figure 2G). Notably, strains with dysfunctional agr showed a significantly higher intracellular persistence than strains with functional agr ( $p$ $=0.050$; Figure $2 \mathbf{H})$. No significant differences were detected between strains of MRSA and MSSA ( $p=0.371$; Figure 2I). The stratified analysis performed to determine the possible interaction of methicillin resistance on agr results showed the same significant trend toward higher intracellular persistence in agr dysfunctional strains both in MSSA and MRSA; however results were not significant in either case probably due to the reduced number of strains $(p=0.267$ and $p=0.667$, respectively), indicating that agr dysfunctional strains were more prone to persist intracellularly regardless of their susceptibility to methicillin. It is worth mentioning that small colony variants (SCV) phenotype was observed in the three CC8 strains and in one CC45 (SA520) and one CC5 (SA80004) in a proportion around $15 \%$ in all cases.

\section{Similar Susceptibility to Human Neutrophil Peptide 1 (hNP-1)}

The results of the susceptibility to hNP-1 obtained for individual strains are shown in Supplementary Figure 3. No significant differences were detected in the percentage of bacterial survival in the presence of hNP-1 peptide between the different CCs, each showing mean survival of around 60\% (CC15 72.0\%, CC30 60.0\%, CC45 64.3\%, CC5 60.3\%, and CC8 58.1\%; p $=0.875$; Figure 3A). Likewise, no significant differences were detected according to $a g r$ functionality, with $a g r$ functional strains exhibiting a mean survival of $65.9 \%$ and agr dysfunctional strains $55.8 \%$ ( $p=0.364$; Figure 3B). Similar bacterial survival was also observed for MRSA and MSSA (54.5 and 65.4\%, respectively, $p=0.454$; Figure $3 \mathrm{C}$ ).

\section{All Strains Formed Biofilm under Static Conditions}

The biofilm formation of the individual strains are shown in Supplementary Figure 4. The biofilm formation assay showed that clinical isolates from all CCs were capable of forming biofilm under static conditions, with mean absorbance levels of around 0.8 at $490 \mathrm{~nm}$ (Figure 4A). No significant differences were detected in the amount of biofilm formed neither between CCs ( $p$ $=0.926$ ) nor between strains with a functional or dysfunctional agr, although it seems to be a trend toward increased absorbance among strains with a dysfunctional $\operatorname{agr}(p=0.089$; Figure 4B). Significant differences in the capacity to form biofilm were detected if strains were grouped according to their methicillin resistance phenotype. Strains of MRSA formed more extensive biofilm than those of MSSA with mean absorbance values of 1.05 and 0.72 , respectively ( $p=0.034$; Figure $4 C$ ). The stratified analysis, performed to determine the possible interaction of agr functionality on these results revealed a significant trend toward higher biofilm formation in MRSA than MSSA in agr functional strains $(p=0.056)$, but not in agr dysfunctional strains $(p=$ 0.800 ) indicating that agr functionality is a confounding factor for these results.

\section{Increased Virulence of CC15 Strains in the Galleria mellonella Model}

Results from analysis of microbial growth kinetics demonstrated that the growth rates between strains of $S$. aureus were similar (Supplementary Figure 5). However, the in vivo G. mellonella model showed differences in virulence between CCs as measured by larvae survival, according to agr functionality and methicillin resistance (Figure 5). The results obtained for individual strains in the virulence assay are shown in Supplementary Figure 6. Larvae inoculated with strains from CC30 had the highest mean survival time (112.53 h; $p<0.001$ for all comparisons), while those inoculated with CC15 had the shortest ( $48.53 \mathrm{~h} ; p<0.001$ for all comparisons), implying an increased virulence of CC15 strains over strains belonging to the other CCs. No significant differences in survival time were demonstrated between larvae infected with CC5 $(77.47 \mathrm{~h})$ and CC8 $(69.33 \mathrm{~h} ; p=0.14)$, CC5 and CC45 (78.04 h; $p=0.76)$, and CC8 and CC45 ( $p=0.1$; Figure 5A).

In this model, strains with a functional agr regulator were found to be more virulent than those with a dysfunctional agr (mean larvae survival time 69.43 and $103.47 \mathrm{~h}$, respectively; $p<$ 0.001; Figure 5B). In addition, MRSA strains were found to be more virulent than MSSA strains (mean larvae survival time were 60.13 and 83.36 h, respectively, $p<0.001$; Figure 5C). For CC5, $\mathrm{CC} 8$, and CC45, infection with MRSA resulted in shorter mean larvae survival time than infection with MSSA from the same CC.

\section{Genomic Analysis of the Virulence Genes Present in CC15 Strains}

In view of the significantly increased virulence of CC15 strains in the in vivo model we characterized their repertoire of virulence genes in an attempt to identify genes or variants that could account for this increased pathogenicity. The genomic comparison of the most relevant virulence genes contained in the analyzed strains revealed differences in the content of genes of strains form different CCs. As expected, in general, all strains from the same CC shared the same virulence genes profile, although some differences in particular genes existed (Table 2). There were no specific virulence genes present in both CC15 strains (SA10009 and SA10014) not found in other CCs that could explain the higher virulence detected for these strains in the in vivo analysis. Remarkably, strains belonging to CC15 did not contain enterotoxins (except sea/sep in SA10009) which were present in almost all the other strains. Similarly, CC15 strains did not contain the sak gene within the Sa3int phage carrying the immune evasion cluster, present in all other strains carrying this phage (all strains except SA80001 and SA70002). The structure of the Sa3int phage, that is located disrupting $h l b$ gene, is in all the other strains $s c n$-chp-sak, but in the case of CC15 strains it only contained $s c n$ and $c h p$ genes. Indeed, when we investigated the DNA microarray results of the whole strains collection we realized that enterotoxins and sak genes were absent in mostly all CC15 strains with only a few particular exceptions. The Panton Valentine leukocidin (PVL) encoded by $l u k F$ and $l u k S$ genes was present only in SA10009. This leukocidin was only present in this strain in the whole collection so it is not common feature 

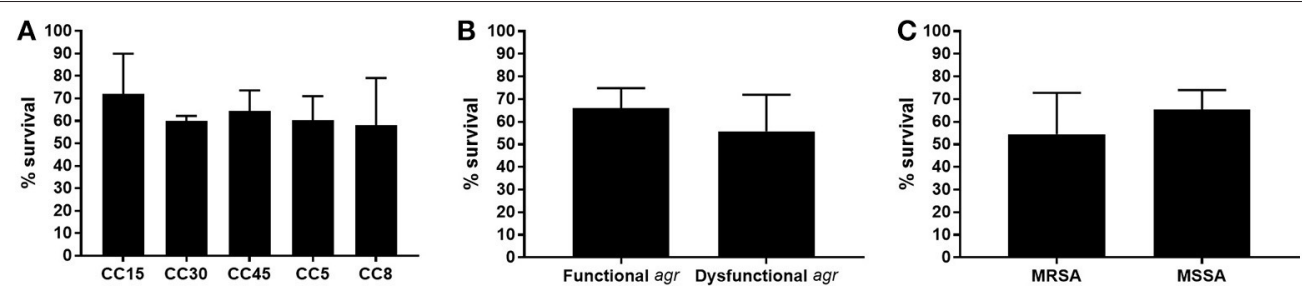

FIGURE 3 | Susceptibility to hNP-1. Susceptibility to hNP-1 of the different CCs (A), according to agr functionality (B), and methicillin resistance (C). The bars and error bars indicate the means and standard deviations for each group, respectively (at least three independent assays per strain). There are no statistically significant differences.
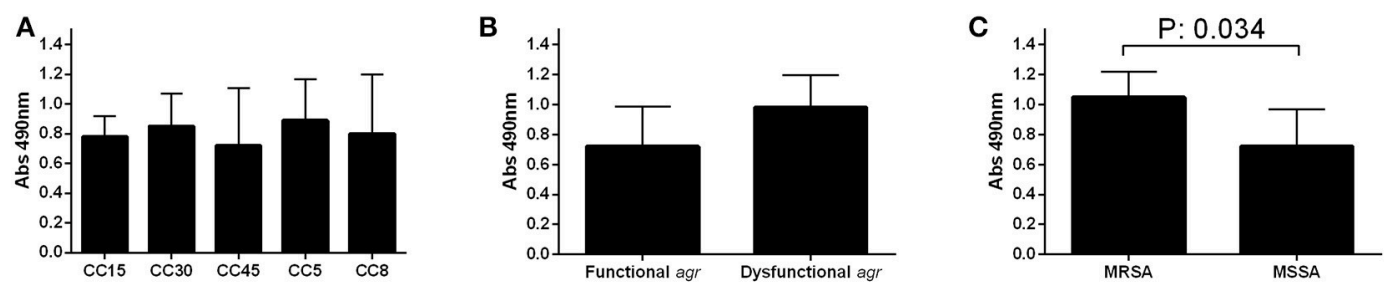

FIGURE 4 | Biofilm formation. Biofilm formation of the different CCs (A), according to agr functionality (B), and methicillin resistance (C). The bars and error bars indicate the means and standard deviations for each group, respectively (at least three independent assays per strain). Only significant $p$-values are shown.
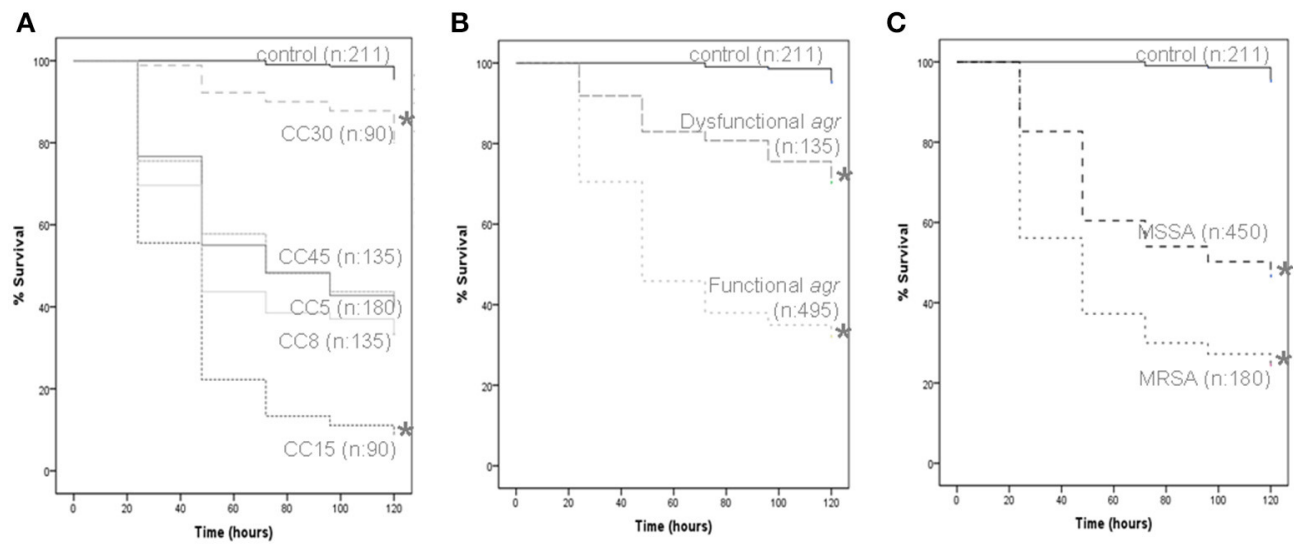

FIGURE 5 | G. mellonella virulence assay. Data from a minimum of three experiments per strain were plotted on Kaplan-Meier survival curves and a log-rank test was used to determine significance. All data grouped according to CC (A), agr functionality (B), and methicillin resistance (C). The number of larvae included in each group is indicated (n). Significance is indicated with asterisks, ${ }^{\star} p<0.001$.

of strains belonging to CC15. It is also worth noting that the Rot regulator was not present in SA10014 strain, but as it is not included in the microarray device we could not study its distribution among all the CC15 strains from the collection.

The virulence genes sequencing analysis in search of variants found in CC15 strains that were not detected in strains from other CCs showed several CC15 specific mutations, most of them resulting in synonymous single nucleotide substitutions (Table 3). No CC15 specific insertions or deletions were found within these genes. Several variants were detected in the agr locus; however this comparison could only be made with CC45 strains that were the only ones with a type II agr locus (sequences could not be aligned with the other agr types found in the remaining
CC5, CC8, and CC30 strains). In general, virulence regulators were highly conserved and we detected only one synonymous mutation at Rot regulator (only present in SA10009). A low number of CC15 specific mutations were detected in hemolysin genes with only one non-synonymous mutation in hla gene. Several CC15 specific variants were detected in leucocidins and proteases, which resulted the most variable subset of virulence genes, with amino acid changing mutations detected in lukE, $s p l A$, and splE. The splA gene showed the highest number of non-synonymous CC15 specific variants with six amino acid changes compared with strains from other CCs. Moreover, two CC15 specific variants, one synonymous and one nonsynonymous were identified in the immune evasion gene chp. 
TABLE 2 | Virulence genes distribution of the 14 analyzed strains.

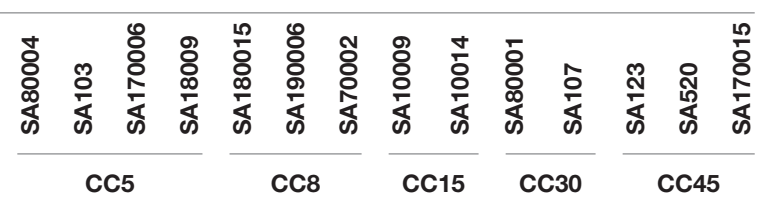

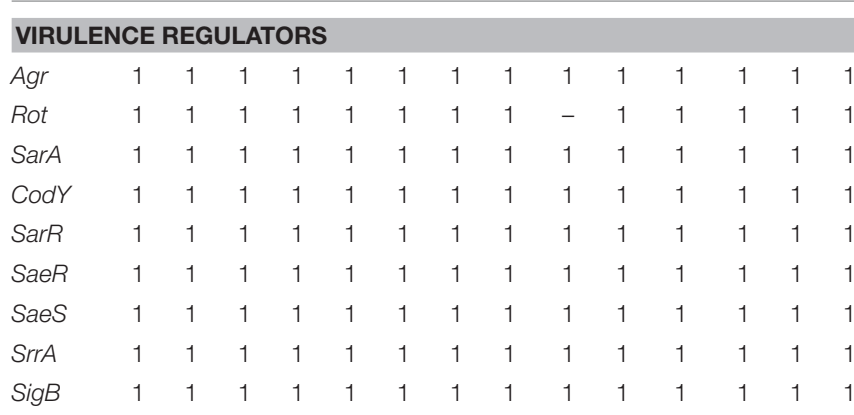

\section{ENTEROTOXINS}

$\begin{array}{lcccccccccccccc}\text { Seg } & 1 & 1 & 1 & 1 & - & 1 & 1 & - & - & 1 & 1 & 1 & 1 & 1 \\ \text { Seo } & 1 & 1 & 1 & 1 & - & 1 & 1 & - & - & 1 & 1 & 1 & 1 & 1 \\ \text { Sen } & 1 & 1 & 1 & 1 & - & 1 & 1 & - & - & 1 & 1 & 1 & 1 & 1 \\ \text { Sea/sep } & 1 & 1 & 1 & 1 & - & 1 & 1 & 1 & - & 1 & 1 & 1 & 1 & 1 \\ \text { Sei } & 1 & 1 & 1 & 1 & - & 1 & 1 & - & - & 1 & 1 & 1 & 1 & 1 \\ \text { Seu } & 1 & 1 & 1 & 1 & - & 1 & 1 & - & - & 1 & 1 & 1 & 1 & 1 \\ \text { Sem } & 1 & 1 & 1 & 1 & - & 1 & 1 & - & - & 1 & 1 & 1 & 1 & 1 \\ \text { Sel } & - & - & - & - & - & - & - & - & - & - & - & 1 & - & - \\ \text { Sec3 } & - & - & - & - & - & - & - & - & - & - & - & 1 & - & - \\ \text { Tst } & - & - & - & - & - & - & - & - & - & 1 & 1 & - & - & - \\ \text { HEMOLYSINS } & & & & & & & & & & & & \\ \text { HIb } & 1 \mathrm{a} & 1^{\mathrm{a}} & 1^{\mathrm{a}} & 1^{\mathrm{a}} & 1^{\mathrm{a}} & 1^{\mathrm{a}} & 1^{\mathrm{a}} & 1^{\mathrm{a}} & 1^{\mathrm{a}} & 1 & 1^{\mathrm{a}} & 1^{\mathrm{a}} & 1^{\mathrm{a}} & 1^{\mathrm{a}} \\ \text { Hla } & 1 & 1 & 1 & 1 & 1 & 1 & 1 & 1 & 1 & 1 & 1 & 1 & 1 & 1 \\ \text { HId } & 1 & 1 & 1 & 1 & 1 & 1 & 1 & 1 & 1 & 1 & 1 & 1 & 1 & 1 \\ \text { hlgB } & 1 & 1 & 1 & 1 & 1 & 1 & 1 & 1 & 1 & 1 & 1 & 1 & 1 & 1 \\ \text { hlgC } & 1 & 1 & 1 & 1 & 1 & 1 & 1 & 1 & 1 & 1 & 1 & 1 & 1 & 1 \\ \text { hlgA } & 1 & 1 & 1 & 1 & 1 & 1 & 1 & 1 & 1 & 1 & 1 & 1 & 1 & 1 \\ \text { Psma1 } & 1 & 1 & 1 & 1 & 1 & 1 & 1 & 1 & 1 & 1 & 1 & 1 & 1 & 1 \\ \text { Psma2 } & 1 & 1 & 1 & 1 & 1 & 1 & 1 & 1 & 1 & 1 & 1 & 1 & 1 & 1 \\ \text { Psma3 } & 1 & 1 & 1 & 1 & 1 & 1 & 1 & 1 & 1 & 1 & 1 & 1 & 1 & 1 \\ \text { Psma4 } & 1 & 1 & 1 & 1 & 1 & 1 & 1 & 1 & 1 & 1 & 1 & 1 & 1 & 1 \\ \text { Psmb1 } & 1 & 1 & 1 & 1 & 1 & 1 & 1 & 1 & 1 & 1 & 1 & 1 & 1 & 1 \\ \text { Psmb2 } & 1 & 1 & 1 & 1 & 1 & 1 & 1 & 1 & 1 & - & - & 1 & 1 & 1\end{array}$

\section{LEUCOCIDINS}

$\begin{array}{lllllllllllllll}\text { lUKE } & 1 & 1 & 1 & 1 & 1 & 1 & 1 & 1 & 1 & - & - & - & - & - \\ \text { lUKD } & 1 & 1 & 1 & 1 & 1 & 1 & 1 & 1 & 1 & - & - & - & - & - \\ \text { luKF } & - & - & - & - & - & - & - & - & - & - & - & - & - & -\end{array}$

lukS

\section{PROTEASES}

$\begin{array}{lllllllllllllll}\text { Aur } & 1 & 1 & 1 & 1 & 1 & 1 & 1 & 1 & 1 & 1 & 1 & 1 & 1 & 1\end{array}$

$\begin{array}{llllllllllllllll}\text { splA } & 1 & 1 & 1 & 1 & 1 & 1 & 1 & 1 & 1 & - & - & - & -\end{array}$

$\begin{array}{lllllllllllllll}\text { splB } & 1 & 1 & 1 & 1 & 1 & 1 & 1 & 1 & 1 & - & - & - & - & - \\ \text { splE } & - & - & - & - & 1 & 1 & - & 1 & 1 & 1 & 1 & - & - & -\end{array}$

\section{IMMUNE EVASION GENES}

$\begin{array}{lllllllllllllllllll}\text { Sak } & 1 & 1 & 1 & 1 & 1 & 1 & - & - & - & - & 1 & 1 & 1 & 1\end{array}$

$\begin{array}{lllllllllllllllll}\text { Scn } & 1 & 1 & 1 & 1 & 1 & 1 & - & - & - & - & 1 & 1 & 1 & 1\end{array}$

$\begin{array}{llllllllllllllll}\text { Chp } & 1 & 1 & 1 & 1 & 1 & 1 & - & - & - & - & 1 & 1 & 1 & 1\end{array}$

1 indicates that the gene is present and - that it is absent in each particular strain. ${ }^{a} \mathrm{HIb}$ truncated by Sa3int phage.

\section{DISCUSSION}

In the present study we analyzed the pathogenic characteristics of representative S. aureus genetic clonal complexes (e.g., CC5, CC8, CC15, CC30, and CC45) that produced endovascular complications following bacteremia to further our understanding about the genetic background implications on the development of this disease. Our results highlight the relevance of evasion of hNP-1 killing and biofilm formation in the endovascular pathogenesis of $S$. aureus because these traits are shared by all genetic backgrounds analyzed. Nevertheless, we detected important differences in the pathogenic behavior of strains from different CCs regarding their adherence to collagen, capacity to interact with the endothelium and virulence in the in vivo model.

Our results indicated that only strains belonging to CC 30 and CC45 were able to bind to collagen. This is likely to be due to the presence of the cna gene, which is known to encode the primary collagen adhesin, in all the strains analyzed belonging to both CC30 and CC45 genetic backgrounds, but not in the other CCs strains analyzed. Indeed when we revised the DNA microarray data from the whole collection of strains we confirmed that cna gene was always present in CC45 and CC30 strains (with only 6 exceptions out of 69 CC30 strains), and it is always absent in CC5, CC8, and CC15 strains (data not shown). The cna gene has previously been studied as a $S$. aureus virulence determinant in endocarditis and other infectious diseases models. Results comparing wild type and cna mutant strains indicated that cna+ strains caused considerable more septic arthritis symptoms in a mice model and outnumbered the mutant strain $24 \mathrm{~h}$ after inoculation in a rat model of infective endocarditis (Patti et al., 1994; Hienz et al., 1996; Nienaber et al., 2011). The results from the present study also suggest that strains that possess this gene, which is associated to particular genetic backgrounds, may take advantage of improved endovascular attachment to establish sites of infection and therefore, further studies in higher models of endovascular complications should be done to further investigate the role of cna gene in this pathogenesis.

Our results demonstrated differences in the interaction of the different CCs with endothelial cells, and appear to indicate higher invasion and intracellular persistence among strains of CC8 than among strains belonging to other CCs. The lack of statistical significance in some of these results may be a consequence of the high standard deviation observed among CC8 group. A rate around $15 \%$ of SCV was observed in some strains in the intracellular persistence experiment (all CC8, 1 CC5, and 1 CC45) probably caused by the use of gentamicin that induces this phenotype (Massey et al., 2001). We detected differences according to agr functionality, with agr dysfunctional strains showing greater adhesion, invasive capacity and persistence than strains with a functional agr; however these differences were only significant for the intracellular persistence experiment. Moreover, the results from the stratified analysis indicated that agr dysfunctional strains were more prone to persist intracellularly, regardless of their susceptibility to methicillin. Fowler et al. (2004) postulated that agr dysfunction can foster development of an intracellular reservoir of microorganisms that contributes to the development 
TABLE 3 | Virulence genes variants especifically detected in CC15 strains.

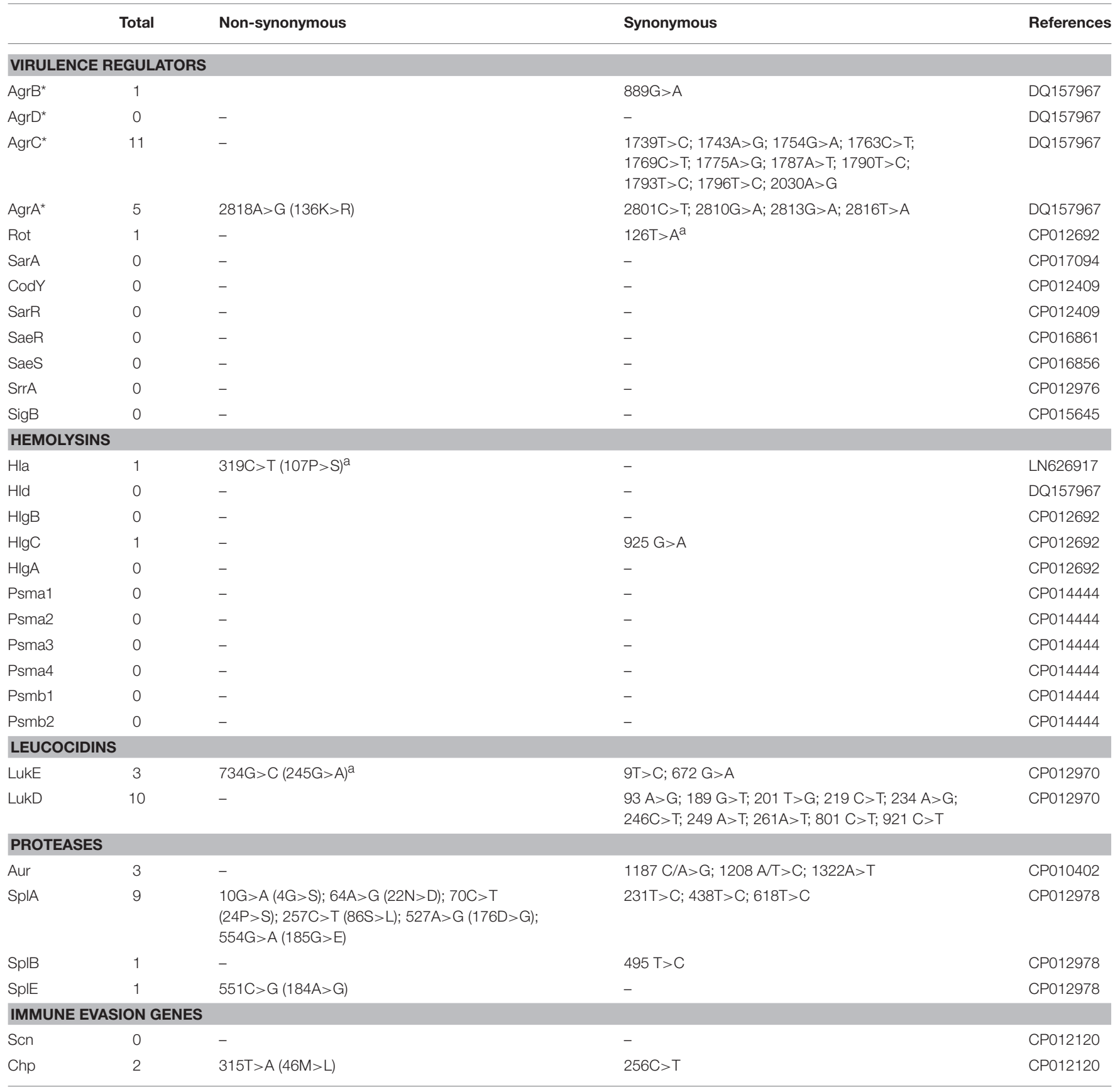

${ }^{*}$ Results from the comparison of CC15 only with CC45 (type II Agr). ${ }^{a}$ Only detected in one of the two CC15 strains.

of infective endocarditis and persistent bacteremia. Moreover, a downregulated and inactive agr system has been previously associated to the intracellularly persisting thymidine-dependent SCV phenotype (Kriegeskorte et al., 2014). In general, we did not observe much difference between strains of MRSA and MSSA in terms of their interaction with endothelial cells.

The survival percentages observed in the presence of hNP-1 were relatively high (58-72\%) in comparison to previous studies that reported mean survival percentages of 41 and $26 \%$ for persistent and resolved bacteremia isolates, respectively (Seidl et al., 2011). Even though we did not detect differences in hNP-1 survival among the different CCs, or according to agr functionality or methicillin resistance phenotype, our results demonstrated that strains causing endovascular complications in general exhibit low susceptibility to hNP-1, which may be indicative of increased ability to evade killing by the host's immune defenses. 
Our results showed that all the strains we studied were able to form biofilm to a similar extent, with no significant differences among the five CCs. The greater biofilm formation detected in MRSA strains in our study was probably influenced by the distribution of agr functionality, because the stratified analysis revealed that these differences were only significant in agr functional strains $(p=0.056)$. Moreover, the associated $a g r$ dysfunction to MRSA strains has been previously reported (Pozzi et al., 2012; McCarthy et al., 2015).

To our knowledge, this is the first report comparing the in vivo virulence of different S. aureus CCs. The G. mellonella in vivo model, has been used to study a wide variety of host-pathogen interactions because there is a correlation between microbial virulence in G. mellonella and that in mammals and because it possesses a complex innate immune system (Peleg et al., 2009; Ramarao et al., 2012). The results of our study in this model revealed significant differences in the behavior of the different CCs. Strains from CC30 exhibited low virulence as measured by low larvae mortality. This agrees with previous studies that also reported significant attenuation of virulence for CC30 in both the G. mellonella and murine sepsis models (SharmaKuinkel et al., 2015). Remarkably, our results indicate high virulence for strains of CC15, with substantial larvae mortality in the first $48 \mathrm{~h}$. Although it has been associated with increased severity of infection and infective endocarditis, there is limited information about the pathogenic mechanisms of CC15 and its involvement in endovascular complications (Fowler et al., 2007; Bouchiat et al., 2015). In agreement with previous reports, we found that strains with a functional $a g r$, associated with an upregulated production of toxins and secreted virulence factors, were significantly more virulent than strains with a dysfunctional agr regulator (Peleg et al., 2009). With regard to methicillin resistance, the G. mellonella model showed that MRSA strains caused significantly more larvae mortality than strains of MSSA. Previous clinical studies have also demonstrated a significantly higher rate of hematogenous complications with MRSA isolates in catheter-associated bacteremia, suggesting that these strains may harbor virulence determinants not found in clonally related MSSA (Fowler et al., 2005, 2007). Recent reports suggest that, while strains of MRSA that harbor SCCmecII exhibit high PBP2a expression, decreased cytolytic toxin production and $a g r$ activity, MRSA strains carrying SCCmecIV have variable PBP2a expression and maintain virulence in the absence of antibiotic, trading agr activity for methicillin resistance upon exposure to beta-lactam antibiotics (Pozzi et al., 2012; Rudkin et al., 2012). This observation would explain our results because all the analyzed MRSA strains were of the SCCmecIV genotype, and would be expected to maintain virulence in the absence of antimicrobial selective pressure as in the conditions of our experiment. The G. mellonella model allowed us to make an initial screening, analyzing 14 strains from five different CCs, and more sophisticated in vivo models should be used to validate the most relevant results.

In an effort to identify the genetic determinants that could be responsible for the increased virulence of CC15 strains in the in vivo model we characterized their repertoire of virulence genes. The analysis of CC15 virulence genes did not allow us to identify any virulence determinant specifically present in CC15 but not in the other CCs, that could be responsible for the higher virulence caused by these strains. Anyway, it is worth mentioning that CC15 strains showed a particular collection of virulence genes in which enterotoxins and the sak immune evasion gene are usually not present. Even though a common characteristic of both CC15 strains explaining their increased virulence could not be detected, the particular strains did present some determinants that could account for this phenotype. In one hand, SA10009 presented the lukFS genes coding for PVL, a two component poreforming exotoxin with an important cytotoxic role on human neutrophils (Löffler et al., 2010), that was not present in any other strain. On the other hand, SA10014 strain did not contain the Rot regulator, that represses virulence genes that encode for exo-toxins, hemolysins, proteases and exo-enzymes (SaïdSalim et al., 2003); therefore its absence could be responsible for an increased virulence. Several CC15 specific variants were detected in the virulence genes analyzed. Overall, leucocidins and proteases showed the highest number of CC15 specific variants. A total of 10 synonymous CC15 specific variants were detected within $l u k D$ gene and two more within $l u k E$ (the nonsynonymous mutation in $l u k E$ is present in only one of the CC15 strains). The lukDE is a bi-component pore-forming leukotoxin targeting and killing neutrophils that had been reported as a major virulence factor involved in bloodstream infections that promotes disease progression via its potent cytotoxic effects on phagocytes recruited to hematogenously-seeded infection sites (Alonzo et al., 2012). These authors reported a 100\% conservation at the amino acid level in a collection of publicly available sequenced $S$. aureus strains. Even though the detected variants were synonymous and low impact at the protein level is expected, further studies would be needed to discard a potential effect of these variants on virulence. Regarding proteases, splA showed the highest number of CC15 specific variants, with a total of six non-synonymous and three synonymous mutations. SplA is one of the serin protease-like proteins that had demonstrated to modulate $S$. aureus physiology and virulence in a rabbit model of pneumonia from which authors concluded that splA may promote invasion and spreading by removing mucin 16 from epithelial cells, what could facilitate infection at multiple body sites (Paharik et al., 2016). The CC15 specific variants detected do not localized in the catalytic or relevant residues described by Stec-Niemczyk et al. (2009) within slpA structure. Therefore, the CC15 splA specific amino acid variants identified should be further investigated to elucidate its potential impact in virulence.

Our results indicated that strains from CC30 showed reduced virulence in the G. mellonella model and higher binding to collagen, in agreement with previous reports that associated the acquisition of genetic variants of agrC, hla, and psma3 genes in this CC with attenuated virulence in animal models and reduced pro-inflammatory potential (Cheung et al., 2014; Sharma-Kuinkel et al., 2015). Conversely, our results indicate that strains from CC15 are highly virulent in the G. mellonella in vivo model, and that this is not due to a greater propensity for biofilm formation, reduced susceptibility to hNP-1, increased capacity to adhere to collagen and endothelial cells, or an enhanced ability to invade and persist intracellularly. The genomic analysis of CC15 
virulence genes, even though identified some specific features of CC15 virulence profile, did not allow us to identify any gene that could be responsible for this increased virulence. Moreover, although several CC15 specific variants were detected in these genes, none of them seems to have a direct impact in virulence. Transcriptomic studies would be needed to further investigate the hitherto unknown pathogenic mechanism(s) responsible for the high larvae mortality caused by these strains of CC15.

We recognize some limitations in the present study. The relatively small number of strains affords a substantial chance for statistical bias and does not allow performing proper multivariate analyses; therefore results should be taken cautiously and larger strain cohorts are needed to validate our findings. However, we performed extensive and time-consuming in vivo and in vitro experiments with all isolates, which cannot be performed automatically at a large scale. In addition, we performed in vitro testing of hNP-1 at sublethal concentrations below those that would be encountered in vivo. We did not identify the genetic factors or underlying pathogenic mechanism(s) responsible for the different behaviors observed among CCs in the in vivo model, and further investigation is required to establish the determinants of these observations. The strengths of the present study include the focus on a specific and well-defined type of infection and outcome that enable drawing firm conclusions. In addition, we selected a representative collection of clinical S. aureus strains from multicenter studies within the most relevant and common CCs causing endovascular complications and performed a variety of in vivo and in vitro experiments to analyze different pathogenic characteristics that might be associated with worse clinical outcome.

\section{CONCLUSIONS}

Despite the fact that all the strains included in the study were known to be capable of causing endovascular complications, our results point out differences in their behavior and/or pathogenic potential. This suggests that the development of endovascular complications can occur through different mechanisms such that each particular CC takes advantage of its specific virulence determinants. These may include higher cytotoxicity as observed with CC15 or increased ability to interact with host endothelium as seen with CC30, CC45, and CC8. Our genomic approach identified some particular CC15 specific features, but transcriptomic analysis would be needed to investigate CC15 pathogenic mechanism in detail. The characterization of the behavior of $S$. aureus most relevant clonal complexes (CCs) causing endovascular complications presented in this work is a needed step before proceeding to more sophisticated models to further understand the etiology of this disease. If the differences detected were confirmed in higher models it would help identifying those patients with poor prognosis and indicate the need to recommend more specific management or therapeutic approaches to the different $S$. aureus CCs according their pathogenic potential.

\section{AUTHOR CONTRIBUTIONS}

Conceived and designed the experiments: DP, FC, and BA; Sample analysis: MM, NL, EV, and NF; Performed the experiments: DP, IM, and PB; Analyzed the data: DP; Draft the manuscript: DP, FC, and EV; Revised the manuscript: NL, NF, $\mathrm{JG}$, and $\mathrm{BA}$; Reviewed and approved the final version of the manuscript: DP, EV, MM, IM, NL, PB, NF, JG, BA, and FC.

\section{FUNDING}

This work was supported by the Health Research Fund (FIS), Department of Health, Spain; Agency for Health Technology Assessment and Research (PI12/01205, PI15/02013, PI12/0179, and PI15/02125) and Instituto de Salud Carlos III, Subdirección General de Redes y Centros de Investigación Cooperativa, Ministerio de Economía y Competitividad, Spanish Network for Research in Infectious Diseases (REIPI RD12/0015 and 0003) and cofunded by the European Regional Development Fund (FEDER).

\section{ACKNOWLEDGMENTS}

We thank the laboratory of Paz de la Torre from Research Center at Hospital Universitario 12 de Octubre for providing endothelial cells.

\section{SUPPLEMENTARY MATERIAL}

The Supplementary Material for this article can be found online at: http://journal.frontiersin.org/article/10.3389/fmicb. 2017.00917/full\#supplementary-material

Supplementary Figure 1 | Adherence to collagen of the 14 analyzed strains individually.

Supplementary Figure 2 | Adhesion, invasion, and intracellular endothelial cells assay of the 14 analyzed strains individually.

Supplementary Figure 3 | Susceptibility to hNP-1 of the 14 analyzed strains individually.

Supplementary Figure 4 | Biofilm formation of the 14 analyzed strains individually.

Supplementary Figure 5 | Growth kinetics of all selected S. aureus strains. Supplementary Figure 6 | G. mellonella virulence array of the 14 analyzed strains individually.

Supplementary Table 1 | General characteristics of S. aureus genomes sequencing and assembly.

\section{REFERENCES}

Alonzo, F. III., Benson, M. A., Chen, J., Novick, R. P., Shopsin, B., and Torres, V. J. (2012). Staphylococcus aureus leucocidin ED contributes to systemic

infection by targeting neutrophils and promoting bacterial growth in vivo. Mol. Microbiol. 83, 423-435. doi: 10.1111/j.1365-2958.2011.07942.x

Aziz, R. K., Bartels, D., Best, A. A., DeJongh, M., Disz, T., Edwards, R. A., et al. (2008). The RAST Server: rapid annotations using 
subsystems technology. BMC Genomics 9:75. doi: 10.1186/1471-216 4-9-75

Bouchiat, C., Moreau, K., Devillard, S., Rasigade, J. P., Mosnier, A., Geissmann, T., et al. (2015). Staphylococcus aureus infective endocarditis versus bacteremia strains: subtle genetic differences at stake. Infect. Genet. Evol. 36, 524-530. doi: 10.1016/j.meegid.2015.08.029

Chaves, F., Garcia-Martinez, J., de Miguel, S., Sanz, F., and Otero, J. R. (2005). Epidemiology and clonality of methicillin-resistant and methicillin-susceptible Staphylococcus aureus causing bacteremia in a tertiary-care hospital in Spain. Infect. Control Hosp. Epidemiol. 26, 150-156. doi: 10.1086/502519

Cheung, G. Y., Kretschmer, D., Duong, A. C., Yeh, A. J., Ho, T. V., Chen, Y., et al. (2014). Production of an attenuated phenol-soluble modulin variant unique to the MRSA clonal complex 30 increases severity of bloodstream infection. PLoS Pathog. 10:e1004298. doi: 10.1371/journal.ppat.1004298

Davis, J., Crampton, S. P., and Hughes, C. (2007). Isolation of Human Umbilical Vein Endothelial Cells (HUVEC). J. Vis. Exp. 3:e183. doi: 10.3791/183

Dayan, G. H., Mohamed, N., Scully, I. L., Cooper, D., Begier, E., Eiden, J., et al. (2016). Staphylococcus aureus: the current state of disease, pathophysiology and strategies for prevention. Expert Rev. Vaccines 15, 1373-1392. doi: 10.1080/14760584.2016.1179583

Enright, M. C., Day, N. P., Davies, C. E., Peacock, S. J., and Spratt, B. G. (2000). Multilocus sequence typing for characterization of methicillin-resistant and methicillin-susceptible clones of Staphylococcus aureus. J. Clin. Microbiol. 38, 1008-1015.

Fernández-Hidalgo, N., Larrosa, N., Pérez-Montarelo, D., Viedma, E., Sáez, C., de Alarcón, A., et al. (2016). "Phenotypic and genotypic risk factors of in-hospital mortality in Staphylococcus aureus infective endocarditis. Preliminary results," in 26th European Congress of Clinical Microbiology and Infectious Diseases (Amsterdam).

Fowler, V. G. Jr., Justice, A., Moore, C., Benjamin, D. K. Jr., Woods, C. W., Campbell, S., et al. (2005). Risk factors for hematogenous complications of intravascular catheter-associated Staphylococcus aureus bacteremia. Clin. Infect. Dis. 40, 695-703. doi: 10.1086/427806

Fowler, Jr. V. G. I., Nelson, C. L., McIntyre, L. M., Kreiswirth, B. N., Monk, A., Archer, G. L., et al. (2007). Potential associations between hematogenous complications and bacterial genotype in Staphylococcus aureus infection. J. Infect. Dis. 196, 738-747. doi: 10.1086/520088

Fowler, V. G., Sakoulas, G., McIntyre, L. M., Meka, V. G., Arbeit, R. D., Cabell, C. H., et al. (2004). Persistent bacteremia due to methicillin-resistant Staphylococcus aureus infection is associated with agr dysfunction and low-level in vitro resistance to thrombin-induced platelet microbicidal protein. J. Infect. Dis. 190, 1140-1149. doi: 10.1086/423145

Ganz, T. (2003). Defensins: antimicrobial peptides of innate immunity. Nat. Rev. Immunol. 3, 710-720. doi: 10.1038/nri1180

Garciarena, C. D., McHale, T. M., Watkin, R. L., and Kerrigan, S. W. (2015). Coordinated molecular cross-talk between Staphylococcus aureus, endothelial cells and platelets in bloodstream infection. Pathogens 4, 869-882. doi: 10.3390/pathogens4040869

Garzoni, C., and Kelley, W. L. (2009). Staphylococcus aureus: new evidence for intracellular persistence. Trends Microbiol. 17, 59-65. doi: 10.1016/j.tim.2008.11.005

Gillaspy, A. F., Lee, C. Y., Sau, S., Cheung, A. L., and Smeltzer, M. S. (1998). Factors affecting the collagen binding capacity of Staphylococcus aureus. Infect. Immun. $66,3170-3178$.

Hienz, S. A., Schennings, T., Heimdahl, A., and Flock, J. I. (1996). Collagen binding of Staphylococcus aureus is a virulence factor in experimental endocarditis. J. Infect. Dis. 174, 83-88. doi: 10.1093/infdis/174.1.83

Kriegeskorte, A., Grubmüller, S., Huber, C., Kahl, B., von Eiff, C., Proctor, RA, et al. (2014). Staphylococcus aureus small colony variants show common metabolic features in central metabolism irrespective of the underlying auxotrophism. Front. Cell. Infect. Microbiol. 4:141. doi: 10.3389/fcimb.2014.00141

Lindsay, J. A., Moore, C. E., Day, N. P., Peacock, S. J., Witney, A. A., Stabler, R. A., et al. (2006). Microarrays reveal that each of the ten dominant lineages of Staphylococcus aureus has a unique combination of surface-associated and regulatory genes. J. Bacteriol. 188, 669-676. doi: 10.1128/JB.188.2.669676.2006

Löffler, B., Hussain, M., Grundmeier, M., Brück, M., Holzinger, D., Varga, G., et al. (2010). Staphylococcus aureus panton-valentine leukocidin is a very potent cytotoxic factor for human neutrophils. PLoS Pathog. 6:e1000715. doi: 10.1371/journal.ppat.1000715

Massey, R. C., Buckling, A., and Peacock, S. J. (2001). Phenotypic switching of antibiotic resistance circumvents permanent costs in Staphylococcus aureus. Curr. Biol. 11, 1810-1814. doi: 10.1016/S0960-9822(01)00507-3

McCarthy, H., Rudkin, J. K., Black, N. S., Gallagher, L., O’Neill, E., and O’Gara, J. P. (2015). Methicillin resistance and the biofilm phenotype in Staphylococcus aureus. Front. Cell. Infect. Microbiol. 5:1. doi: 10.3389/fcimb.2015.00001

Monecke, S., Berger-Bachi, B., Coombs, G., Holmes, A., Kay, I., Kearns, A., et al. (2007). Comparative genomics and DNA array-based genotyping of pandemic Staphylococcus aureus strains encoding panton-valentine leukocidin. Clin. Microbiol. Infect. 13, 236-249. doi: 10.1111/j.1469-0691.2006.01635.x

Monecke, S., Slickers, P., and Ehricht, R. (2008). Assignment of Staphylococcus aureus isolates to clonal complexes based on microarray analysis and pattern recognition. FEMS Immunol. Med. Microbiol. 53, 237-251. doi: 10.1111/j.1574-695X.2008.00426.x

Naber, C. K. (2009). Staphylococcus aureus bacteremia: epidemiology, pathophysiology, and management strategies. Clin. Infect. Dis. 48(Suppl. 4), S231-S237. doi: 10.1086/598189

Nienaber, J. J., Sharma Kuinkel, B. K., Clarke-Pearson, M., Lamlertthon, S., Park, L., Rude, T. H., et al. (2011). Methicillin-susceptible Staphylococcus aureus endocarditis isolates are associated with clonal complex 30 genotype and a distinct repertoire of enterotoxins and adhesins. J. Infect. Dis. 204, 704-713. doi: 10.1093/infdis/jir389

Paharik, A. E., Salgado-Pabon, W., Meyerholz, D. K., White, M. J., Schlievert, P. M., and Horswill, A. R. (2016). The Spl serine proteases modulate Staphylococcus aureus protein production and virulence in a rabbit model of pneumonia. mSphere 12:1. doi: 10.1128/mSphere.00208-16

Patti, J. M., Bremell, T., Krajewska-Pietrasik, D., Abdelnour, A., Tarkowski, A., Rydén, C., et al. (1994). The Staphylococcus aureus collagen adhesin is a virulence determinant in experimental septic arthritis. Infect. Immun. 62, $152-161$.

Peacock, S. J., Moore, C. E., Justice, A., Kantzanou, M., Story, L., Mackie, K., et al. (2002). Virulent combinations of adhesin and toxin genes in natural populations of Staphylococcus aureus. Infect. Immun. 70, 4987-4996. doi: 10.1128/IAI.70.9.4987-4996.2002

Peleg, A. Y., Monga, D., Pillai, S., Mylonakis, E., Moellering, R. C., and Eliopoulos, G. M. (2009). Reduced susceptibility to vancomycin influences pathogenicity in Staphylococcus aureus infection. J. Infect. Dis. 199, 532-536. doi: $10.1086 / 596511$

Pozzi, C., Waters, E. M., Rudkin, J. K., Schaeffer, C. R., Lohan, A. J., Tong, P., et al. (2012). Methicillin resistance alters the biofilm phenotype and attenuates virulence in Staphylococcus aureus device-associated infections. PLoS Pathog. 8:e1002626. doi: 10.1371/journal.ppat.1002626

Proctor, R. A., von Eiff, C., Kahl, B. C., Becker, K., McNamara, P., Herrmann, M, et al. (2006). Small colony variants: a pathogenic form of bacteria that facilitates persistent and recurrent infections. Nat. Rev. Microbiol. 4, 295-305. doi: 10.1038/nrmicro1384

Ramarao, N., Nielsen-Leroux, C., and Lereclus, D. (2012). The insect Galleria mellonella as a powerful infection model to investigate bacterial pathogenesis. J. Vis. Exp. 70:e4392. doi: 10.3791/4392

Richards, R. L., Haigh, R. D., Pascoe, B., Sheppard, S. K., Price, F., Jenkins, D., et al. (2015). Persistent Staphylococcus aureus isolates from two independent cases of bacteremia display increased bacterial fitness and novel immune evasion phenotypes. Infect. Immun. 83, 3311-3324. doi: 10.1128/IAI.00255-15

Rigby, K. M., and DeLeo, F. R. (2012). Neutrophils in innate host defense against Staphylococcus aureus infections. Semin. Immunopathol. 34, 237-259. doi: 10.1007/s00281-011-0295-3

Rudkin, J. K., Edwards, A. M., Bowden, M. G., Brown, E. L., Pozzi, C., Waters, E. M., et al. (2012). Methicillin resistance reduces the virulence of healthcare-associated methicillin-resistant Staphylococcus aureus by interfering with the agr quorum sensing system. J. Infect. Dis. 205, 798-806. doi: 10.1093/infdis/jir845

Saïd-Salim, B., Dunman, P. M., McAleese, F. M., Macapagal, D., Murphy, E., McNamara, P. J., et al. (2003). Global regulation of Staphylococcus aureus genes by Rot. J. Bacteriol. 185, 610-619. doi: 10.1128/JB.185.2.610-619.2003

San-Juan, R., Viedma, E., Chaves, F., Lalueza, A., Fortún, J., Loza, E., et al. (2016). High MICs for vancomycin and daptomycin and complicated catheter-related 
bloodstream infections with methicillin-sensitive Staphylococcus aureus. Emerg. Infect. Dis. 6, 1057-1066. doi: 10.3201/eid2206.151709

Seidl, K., Chen, L., Bayer, A. S., Hady, W. A., Kreiswirth, B. N., and Xiong, Y. Q. (2011). Relationship of agr expression and function with virulence and vancomycin treatment outcomes in experimental endocarditis due to methicillin-resistant Staphylococcus aureus. Antimicrob. Agents Chemother. 55, 5631-5639. doi: 10.1128/AAC.05251-11

Sharma-Kuinkel, B. K., Mongodin, E. F., Myers, J. R., Vore, K. L., Canfield, G. S., Fraser, C. M., et al. (2015). Potential influence of Staphylococcus aureus clonal complex 30 genotype and transcriptome on hematogenous infections. Open Forum Infect. Dis. 2:ofv093. doi: 10.1093/ofid/ ofv093

Sinha, B., and Fraunholz, M. (2010). Staphylococcus aureus host cell invasion and post-invasion events. Int. J. Med. Microbiol. 300, 170-175. doi: 10.1016/j.ijmm.2009.08.019

Stec-Niemczyk, J., Pustelny, K., Kisielewska, M., Bista, M., Boulware, K. T., Stennicke, H. R., et al. (2009). Structural and functional characterization of SplA, an exclusively specific protease of Staphylococcus aureus. Biochem. J. 419, 555-564. doi: 10.1042/BJ20 081351
Waterhouse, J. C., and Russell, R. R. (2006). Dispensable genes and foreign DNA in Streptococcus mutans. Microbiology 152(Pt 6), 1777-1788. doi: 10.1099/mic.0.28647-0

Xiong, Y. Q., Fowler, V. G., Yeaman, M. R., Perdreau-Remington, F., Kreiswirth, B. N., and Bayer, A. S. (2009). Phenotypic and genotypic characteristics of persistent methicillin-resistant Staphylococcus aureus bacteremia in vitro and in an experimental endocarditis model. J. Infect. Dis. 199, 201-208. doi: $10.1086 / 595738$

Conflict of Interest Statement: The authors declare that the research was conducted in the absence of any commercial or financial relationships that could be construed as a potential conflict of interest.

Copyright (C) 2017 Pérez-Montarelo, Viedma, Murcia, Muñoz-Gallego, Larrosa, Brañas, Fernández-Hidalgo, Gavaldà, Almirante and Chaves. This is an open-access article distributed under the terms of the Creative Commons Attribution License (CC $B Y)$. The use, distribution or reproduction in other forums is permitted, provided the original author(s) or licensor are credited and that the original publication in this journal is cited, in accordance with accepted academic practice. No use, distribution or reproduction is permitted which does not comply with these terms. 